As a result of this, collaborative work was undertaken with Radiology colleagues to determine the optimal and safest imaging protocol for managing patients conservatively.

Furthermore, a Lateral Skull Base Multidisciplinary Team discussion meeting and clinic were conceived and delivered, dramatically reducing patient waiting times for clinic appointments and results. This has culminated in the Lateral Skull Base service being awarded the 2019 Leads Teaching Hospitals 'Time to Shine' award for Improving Quality and Safety.

\section{THE PATIENT-CENTRED MANAGEMENT (PCM) ACADEMY - A CUSTOMIZED LEAN HOSPITAL TRAINING}

Sabine Kressmann, Kurt Aldorf, med Katharina Rüther-Wolf. University Hospital Basel, Switzerland

\subsection{6/leader-2019-FMLM.79}

If you want to change to a Lean hospital with the aim of patient centricity, the main factor is 'culture'. During a Lean transformation, it is not only key to properly train the staff in Lean principles, but also to trigger a cultural change and thus ensuring the sustainability of the Lean transformation. One of the major aspects to achieve the latter is to educate a view for i) processes and ii) the full cycle of care. To allow for an equal level of understanding of Lean principles across the hospital and to embark everyone on the Lean journey, a customized 'Lean Hospital Training'- The PCM Academy was developed. Over the course of five levels, employees across all professions are slowly introduced to the main Lean tools. Thanks to an interactive, didactical approach attendees learn through simulations and role-plays. In addition, attendees value the fact to learn and work on their own real live and day-to-day examples/projects. Starting from level 3, attendees have homework and their own little projects between the course days. The modules are trained by interprofessional coaches, who still work in the clinic in addition to their role in the PCM Academy, either as doctor, nurse or project manager. Our approach is 'as little (slides) as possible and as much as necessary'. Activities and simulations animate attendees to be in an active 'doing' mode and not passive 'listening' mode. By working on their own examples, attendees can immediately implement some of the changes in their daily work. Over the past 2 years we did observe a clear increase in demand for the PCM Academy courses. Also, the main Lean tools like, 'gemba', 'kaizen' and 'huddle', are not only familiar but also lived by most of the staff. By empowering the staff across all professions and hierarchical positions, we aim to see a clear increase in i) Patient centricity, ii) Trigger of cultural change and iii) Increased sustainability.

\section{GENDER DISPARITY IN ACHIEVING SENIORITY IN MEDICAL EDUCATION: THE GLASS CEILING IS YET TO BE BROKEN}

Luthfun Nessa* Julie Browne*. Cardiff University School of Medicine

10.1136/leader-2019-FMLM.80

Background The number of females in senior and leadership positions in Medical Education is disproportionate to the female intake at the undergraduate and postgraduate level. This has been a slowly recognised phenomenon and 170 years after the first female medical graduate, little remains changed in achieving gender equity at the senior level.

Objective To identify what factors specific to women prevents their career progression in Medical Education.

Method Email invitations were sent to female members of the Academy of Medical Education leading to seven semi-structured telephone interviews. Qualitative analysis of six interview transcripts were carried using Nvivo11.

Common themes from all informants were collated and studied.

Population/Participants The final cohort consisted of two deans of postgraduate medicine, one deputy dean of undergraduate medicine, one managing director, one clinical and one honorary lecturer.

Findings The most significant factors that negatively affect career progression include: 1)Design of the Institution infrastructure 2) Work-Life balance 3) Lack of role models and mentors 4) Networking opportunities. Other common themes were intrinsic gender bias against women due to 'female' personality traits, lack of self-confidence and not seeking professional recognition as commonly as men.

Conclusion Interviewees expressed the importance of a support system which enables adequate work life management. For all respondents expressed that having a supportive husband was key to their success, a theme which has not been previously identified in the literature. This calls for a change in both the work place and at home as a society we must realise that the domestic duties predominantly placed upon women, halts their progression along the career ladder. If changes are not made soon, the work force will be deprived of a very educated cohort.

\section{LEADING THE WAY TOWARDS PARITY OF ESTEEM}

${ }^{1}$ Hanad Ahmed, ${ }^{2}$ Arion Alexopolous, ${ }^{2}$ Alex Compton, ${ }^{3}$ Rita Otto, ${ }^{4}$ Gabrielle Sanders, ${ }^{2}$ Yusuf Patel. ${ }^{1}$ University of Southampton, UK; ${ }^{2}$ Imperial College London, UK; ${ }^{3}$ Cardiff University, UK; ${ }^{4}$ King's College London, UK; ${ }^{5}$ Imperial College London, UK

\subsection{6/leader-2019-FMLM.81}

Mental health (MH) problems account for more than a quarter of the UK's disease burden, with an estimated economic cost of $£ 105$ billion a year. Three quarters of chronic $\mathrm{MH}$ problems start before age 18 . With the absolute rise in population mental ill-health, this problem is only set to grow. As the economic cost grows, this is an issue that will affect allnot just those with mental ill-health.

Assessment of the current situation and government plans identified some of the causes leading to poor Mental Health care. Using the Pareto Principle to stratify the causes, three key areas were identified: Children \& Adolescents, Community Training and Data Collection \& Research. The findings of this work have been presented to students and academics at Imperial College London.

Within each of these three areas, recommendations for the next five years offer an opportunity for healthcare professionals, primary care workers in particular, to support a healthier population and greater patient satisfaction. Implementation of these recommendations requires careful management and strong vision and leadership from healthcare professionals. 